\title{
Felicidad, instrumentalidad y expresividad en dos muestras latinoamericanas: México y Bolivia
}

\author{
Rolando Díaz-Loving \\ Pedro Wolfgang Velasco-Matus \\ Sofía Rivera-Aragón \\ Universidad Nacional Autónoma de México \\ Ciudad de México, México
}

\begin{abstract}
Recibido: 15 de noviembre del 2017 / Aceptado: 3 de enero del 2018 doi: https://doi.org/10.26439/persona2018.n021.1989
\end{abstract}

El presente trabajo tuvo dos objetivos: 1) estimar el efecto que tienen el sexo y el lugar de origen en los niveles de felicidad y rasgos de género, y 2) evaluar la relación entre felicidad y rasgos de género en una muestra de hombres y mujeres de México y Bolivia. Participaron 998 personas: 583 bolivianos (293 hombres, 290 mujeres) y 425 mexicanos (208 hombres, 207 mujeres), de entre 18 y 64 años. Para evaluar felicidad, se utilizó la Escala de Felicidad de Alarcón (2006), y para evaluar instrumentalidad-expresividad, la Escala de Dimensiones Atributivas de Instrumentalidad y Expresividad (Edaie). Se llevaron a cabo anova (Analysis of Varience) $2 \times 2$ [Sexo (hombre-mujer) x lugar de origen (Bolivia-México)] para tasar el efecto de interacción sobre los tres factores de felicidad $y$ cuatro de género, y correlaciones producto-momento de Pearson entre estos. Los análisis de varianza muestran efectos de interacción de sexo x lugar de origen solo en dos factores de género; mientras que las correlaciones muestran que instrumentalidad y expresividad positiva están asociados con buenos niveles de felicidad. Los resultados se discuten a la luz de características culturales, como el colectivismo-individualismo, el lugar de origen, el sexo de las personas y teorías de bienestar-felicidad.

felicidad / género / cultura / sexo / colectivismo

Correo electrónico: rdiazl@unam.mx 


\section{Happiness, Instrumentality and Expressiveness in Two Latin American Samples: Mexico and Bolivia}

This study had two objectives: (1) to assess the effect that sex and place of origin have on levels of happiness and gender traits, and (2) to evaluate the relationship between happiness and gender traits on men and women from Mexico and Bolivia. The study population consisted of 998 volunteers: 583 Bolivian (293 men, 290 women) and 425 Mexicans (208 men, 207 women) aged between 18 and 64 years. To assess happiness, we used Alarcón's Happiness Scale (2006). To assess gender, we used the Instrumentality and Expressiveness Attributional Dimensions Scale (EDAIE). A two-way analysis of variance (ANOVA) [sex (man-woman) x place of origin (Bolivia-Mexico)] was used to evaluate the interaction effect on three happiness and four gender factors. The Pearson productmoment correlation coefficient was used to measure the relationship between them. The ANOVA showed interaction effects of sex $x$ place of origin only on two gender factors. The Pearson correlation coefficient showed that positive instrumentality and expressiveness are associated with optimal levels of happiness. The results are discussed in terms of cultural characteristics, such as individualism-collectivism, place of origin, sex, and happiness/ well-being theories. 


\section{INTRODUCCIÓN}

La felicidad y cómo alcanzarla son temas que han intrigado a las personas desde la antigüedad. En el ámbito de la Psicología, durante la primera mitad del siglo $\mathrm{xx}$, se llevaron a cabo esfuerzos importantes por comprender la felicidad. Típicamente, las investigaciones fueron de corte cualitativo, con una meta muy particular: obtener una descripción universal de la persona feliz (Diener, Suh, Lucas y Smith, 1999). Bajo esa línea, para los sesenta, Wilson (1967) presentó un metaanálisis que recopilaba los hallazgos de estudios que se habían realizado en torno a la felicidad. Sobre la base de los datos que se tenían a la fecha, él llegó a la conclusión de que una persona feliz es una persona "joven, saludable, bien pagada, extrovertida, optimista, libre de preocupaciones, religiosa, casada, con buena autoestima, buena moral laboral, aspiraciones modestas de vida y con una buena inteligencia" (p. 294). Para Wilson, el sexo no tenía relación significativa con la felicidad.

Hoy en día, lograr un consenso respecto a la definición de felicidad no es tarea fácil, pues tiende a considerarse que felicidad, bienestar subjetivo, calidad de vida, satisfacción y balance afectivo/hedónico pueden utilizarse como sinónimos (Diener, 2009; Palomar, 2005; Veenhoven, 2005). Las premisas para tal sinonimia son las siguientes: 1) si una persona está satisfecha con su vida y tiene una calidad de vida aceptable/óptima, entonces dicha persona debe ser feliz, y 2) las mediciones de respuestas emocionales, de afecto y juicios globales y específicos de calidad de vida han correlacionado entre sí de manera sustancial, a pesar de ser entendidos y evaluados por separado; por lo que se asume que pudieran tener componentes subyacentes suficientemente similares (Kozma y Stones, 1983).

De acuerdo con Alarcón (2000, 2006), la felicidad es un estado afectivo de satisfacción plena que experimenta un individuo en posesión de un bien deseado/anhelado. El autor también acota algunas propiedades de la conducta feliz (Alarcón, 2006): 1) es un sentimiento de satisfacción que vive una persona, y solo ella, en su vida interior. Esto es, el componente subjetivo individualiza la felicidad entre las personas; 2) se trata de un estado, por lo cual hay que considerar la estabilidad temporal de la felicidad: puede ser duradera o perecible; 3) la felicidad supone la posesión de un bien. Este bien deseado es lo que eventualmente hace feliz a una persona; 4) el bien o bienes que generan felicidad son de naturaleza variada. Puede tratarse de bienes materiales, éticos, estéticos, psicológicos, religiosos, sociales, etcétera. Esto implica que los bienes poseen valencias positivas que las personas les otorgan, lo cual infiere que un bien deseado no necesariamente posee un valor intrínseco, y que un mismo bien no siempre genera felicidad en todos los individuos, y 5) el momento histórico y una sociocultura determinada conllevan a la coincidencia del deseo de algún bien o bienes; así, se llega a una noción de felicidad colectiva. 
Desde hace algún tiempo, las diferencias por sexo en niveles de felicidad no son concluyentes. Muchas investigaciones reportan diferencias mínimas en los referidos a hombres y mujeres cuando se evalúan los niveles de satisfacción y felicidad (Andrews y Withey, 1976; Diener, 2009) y, a pesar de que las diferencias no son significativas, son las mujeres quienes generalmente presentan menores niveles de satisfacción, felicidad y bienestar (Fujita, Diener y Sandvik, 1991). Según Diener y Diener (2009) y Triandis (2000), las diferencias pueden deberse a factores asociados a la libertad, el estatus, recursos y poder; que en gran parte, se encuentran determinados por factores socioculturales.

Biswas-Diener, Vitterso y Diener (2009) añaden que las variaciones culturales en niveles de felicidad están asociadas no solo al sexo de las personas sino a factores, como las normas culturales específicas y el acceso a ciertos recursos sociales y materiales. Los autores además señalan que la cultura determina en cada sexo las expectativas locales y los estándares para evaluar diferentes dominios de la vida. También, Diener y Diener (1996) notaron que en culturas colectivistas parece existir cierta presión por socializar y expresar emociones, de manera que el funcionamiento social sea natural y fluido; lo que, a su vez, lleva a que en este tipo de culturas la gente sea socializada a ser, expresarse y actuar feliz.

Existe evidencia (Tov y Diener, 2009) de que diferentes culturas varían en los correlatos que presentan variables como el bienestar subjetivo y la felicidad. Uno de los hallazgos más recurrentes es que las variaciones culturales pueden marcar la diferencia en niveles de satisfacción de los individuos. Autores como Suh, Diener y Updegraff (2008) atribuyen estas variaciones a dos causas: el colectivo cultural (componente social) y los cambios culturales personales.

A todo esto debe sumársele que una de las dimensiones de la personalidad que recientemente ha sido asociada con la felicidad y el bienestar de las personas es el género. Por ejemplo, Diener (2009) menciona que, desde hace algún tiempo, las características andróginas (exhibir características masculinas $\mathrm{y}$ femeninas de personalidad) están asociadas con mayores niveles de felicidad, lo cual es congruente con los hallazgos de Allen-Kee (1980), Díaz-Loving, Rivera y Velasco (2012), Velasco (2015) y O'Sullivan (1980), entre otros.

Generalmente hacia los tres años de edad, los niños desarrollan una constancia de género (Campbell, 1993); esto es, cierta comprensión de que el género es relativamente permanente, de manera que hombres y mujeres durante la infancia saben que el ser femenino (niñas) es diferente al ser masculino (niños), y que eso no puede variar. Por ello desarrollan una motivación interna para adquirir las características necesarias que les permitan ser competentes en el sexo que les corresponde. Fernández (1996) menciona que esta tarea de corresponder continúa vigente a lo largo de toda la 
vida, y atraviesa diferentes etapas o estadios críticos. Entre los siete y once años existe un proceso de flexibilidad-rigidez de género; en otras palabras, se le da mucha importancia a seguir los comportamientos estipulados por la sociedad en torno a lo masculino y lo femenino. Entre los once y veinte años surge un ajuste reflexivo, por lo que la persona puede presentar una mayor diversificación de rasgos y comportamiento. Finalmente, entre los 20 y 50 se espera que los jóvenes y adultos presenten una realización clara de los roles de género. Así, se desea que las personas se apeguen con mayor intensidad, conforme crecen, a una serie de cosmovisiones sobre lo masculino y lo femenino (Díaz-Loving, 2008).

Conceptualmente, los roles de género son expectativas y creencias populares sobre las actividades, rasgos, características y atributos que distinguen a los hombres de las mujeres, producto de estereotipos socialmente establecidos, adaptados y aceptados para cada entidad genérica (Rocha, 2004). De esta manera, los rasgos de instrumentalidad y expresividad constituyen dimensiones atributivas de personalidad, que son entendidas como aspectos globales del autoconcepto que tienen hombres y mujeres, y que se componen de características normativas para cada género (Díaz-Loving, Rocha y Rivera, 2007; Spence, 1993). Originalmente, masculinidad y feminidad fueron entendidas como extremos opuestos de un continuo. Por ende, las diferencias en atribuciones y roles asignados a cada sexo se extrapolaban al hecho de que cada uno pertenece a mundos diferentes y complementarios, lo que dio origen a los estereotipos de género (Díaz-Loving et al., 2012; Jayme y Sau, 2004). Dichos estereotipos constituyen creencias exageradas, asociadas a cada sexo, e indican cómo deben comportarse hombres y mujeres. Desde esta perspectiva socioculturalmente estereotipada, la masculinidad, en línea con el estereotipo dominante, está asociada al logro, competitividad, autonomía, dominio, racionalidad, resistencia, entre otras características; mientras que la feminidad implica aspectos como la sumisión, dependencia, afectividad y otros roles asociados al cuidado de los otros (Díaz-Loving et al., 2007). Las personas pueden ser femeninas, masculinas o bien indiferenciadas o andróginas (Bem, 1981), lo cual implica que pueden presentar un alto grado de estereotipamiento y rigidez alrededor de su género, y comportarse de forma sumamente tradicional; o bien, pueden alejarse de dichos parámetros.

Según Alarcón (2002), las fuentes de la felicidad son de distintos orígenes; por ejemplo, pueden deberse a factores étnicos, económicos, religiosos, estéticos, sociales, biológicos, materiales e ideales. Asimismo, algunos autores (Díaz-Loving et al., 2012; Matud, Rodríguez, Marrero y Carballeira, 2002) enfatizan cómo algunos factores socio-psicológicos tienen impacto en la masculinidad y feminidad de las personas, y cómo esto eventualmente puede determinar el nivel de bienestar y felicidad de las personas (Díaz-Loving et al., 2012; Velasco, 2015).

Debido a que la cultura sufre transformaciones (Pozos, 2012; Tov y Diener, 
2009; Velasco, 2015), impacta y modifica la manera en que se integran y conforman diferentes estereotipos, incluyendo aquellos vinculados con la instrumentalidad (características ligadas a la masculinidad) y la expresividad (características relacionadas con la feminidad). Esta transformación cultural permite la transformación de lo que originalmente se concebía como dos polos extremos (masculino frente a femenino) en atributos de personalidad autónomos, independientes, que pueden coexistir en cada individuo sin importar si es hombre o mujer (Díaz -Loving et al., 2007, 2012). Así, las personas pueden ser consideradas masculinas, femeninas, andróginas (presencia de masculinidad y feminidad) o indiferenciados (ausencia de masculinidad y feminidad) (Bem, 1981), dependiendo de la rigidez con que cada persona maneje en su vida los estereotipos de género, y se aleje o acerque a cada parámetro.

Diversas investigaciones (Bem, 1993; Díaz-Loving et al., 2007, 2012; Green y Kenrick, 1994; Hegelson y Fritz, 1999; Moen et al., 1995; Matud et al., 2002; Rocha, 2008; Velasco, 2015) dejan entrever que hombres y mujeres pueden llegar a adoptar conductas y estilos de vida que determinan su estabilidad personal, sus niveles de satisfacción, su desarrollo personal, madurez intelectual, relaciones interpersonales, su felicidad, entre otras cosas, a partir de la transformación cultural. Rocha (2008) reporta hallazgos en los que, por una parte, la permanencia en rasgos, roles y actitudes tradicionales conlleva tanto a hombres como mujeres a la experiencia de cierto malestar, que se ve reflejado en el tipo de estados anímicos reportados. Sin embargo, ante la adopción de roles y rasgos no convencionales ni estereotipados también se hace presente este malestar. Es posible que aunque hombres y mujeres experimentan mayor bienestar general cuando incorporan actividades y rasgos no tradicionales, algunos de estos conlleven a un estado anímico de malestar.

El estudio de Rocha (2008) destaca que, aparentemente, sigue existiendo una presión social fuerte en torno a que hombres y mujeres lleven a cabo actividades altamente estereotipadas. Las actividades y rasgos asociados tradicionalmente a cada género conllevan un significado positivo o negativo que facilita el hecho de que ante ciertos roles o características, ambos sexos experimenten malestar, tal como otros autores lo han señalado (Moen et al., 1995). En este sentido, aun si un rol es parte de un estereotipo, dados los cambios sociales e ideológicos actuales, es factible que ambos géneros contrasten los beneficios y costos de un rol de esta naturaleza; por lo que, en este sentido, las connotaciones afectivas de los estereotipos resultan relevantes como consideración ante la posibilidad de que las personas híbridas en cuestión de rol de género no experimenten malestar.

De este modo, a partir de la literatura revisada, los objetivos de este trabajo fueron: 1) evaluar el efecto que tienen el sexo y el lugar de origen en los niveles de felicidad y rasgos de género, y 2) evaluar la relación entre felicidad y rasgos de género en una muestra latinoamericana. 


\section{Método}

\section{Participantes}

Participaron 998 voluntarios de dos ciudades latinoamericanas; 583 participantes eran de Santa Cruz, Bolivia y 425 de Morelos, México. De los 583 bolivianos, 293 eran hombres y 290 , mujeres, todos con edades de entre veinte y cincuenta años $(\mathrm{M} .=34.2$, D.E. $=9.73)$. El $69.9 \%$ se encontraba en una relación de pareja, y las escolaridades con mayor porcentaje fueron técnico/bachillerato $(28.5 \%)$ y licenciatura $(34 \%)$. En lo que respecta a los 425 mexicanos, 208 eran hombres y 207 , mujeres, todos con edades de entre dieciocho y sesentaicuatro años (M. $=23.86$, D.E. $=9.2)$. El $43.1 \%$ se encontraba en una relación de pareja, y las escolaridades con mayor porcentaje fueron secundaria $(58.1 \%$ ) y técnico/bachillerato $(31.8 \%)$.

\section{Instrumentos}

Se utilizó la Escala de Felicidad de Alarcón (2006), que contiene veintisiete reactivos distribuidos en cuatro dimensiones: sentido positivo de la vida, satisfacción con la vida, realización personal y alegría de vivir. La escala, en su versión original, fue validada en una muestra de Lima, Perú, y reportó tener un alfa global de 0.9 y $49.79 \%$ de la varianza total explicada. Para este trabajo, se utilizó una configuración de tres dimensiones (satisfacción, infelicidad y optimismo), que considera 26 de los reactivos originales (se eliminó el reactivo 13) y que explica $50.4 \%$ de varianza total para la muestra mexicana y $51.7 \%$ para la boliviana. Para ambas muestras, esta nueva configuración de la escala presenta un alfa de Cronbach superior a 0.7.

Para evaluar rasgos de género, se utilizó la Escala de Dimensiones Atributivas de Instrumentalidad y Expresividad (Edaie, Díaz-Loving, Rocha y Rivera, 2007). Originalmente, la escala consta de 117 adjetivos con formato de respuesta tipo Likert (1-nada, 5-muchísimo), que se distribuyen en once factores. No obstante, para este trabajo, se utilizó una versión de 55 reactivos agrupados en cuatro factores (instrumentalidad positiva, instrumentalidad negativa, expresividad positiva y expresividad negativa). La configuración de instrumentalidad positiva y negativa explicó $39.9 \%$ de varianza para México y $38.3 \%$ para Bolivia; mientras que la configuración de expresividad positiva y negativa alcanzó $45.4 \%$ de varianza para México y $43.9 \%$ para Morelos. Los índices alfa de Cronbach son superiores a 0.8 para todos los factores, en ambas muestras.

\section{Resultados}

Para evaluar el efecto que tienen el sexo y lugar de origen sobre los tres factores de felicidad y los cuatro factores de género, se llevó a cabo un anova $2 \times 2$, considerando para sexo las categorías de hombre y mujer y para lugar de origen, Bolivia y México.

En cuanto a instrumentalidad positiva, no se observó efecto de interacción $[F(1,933)=0.008, p>0.05]$ ni principal de sexo $[F(1,933)=3.26, p>0.05]$; sin embargo, sí hubo efecto principal por lugar de origen 
$[F(1,933)=27.24, p<0.05]$. Las medias indican que la muestra de Bolivia (M.=4, D.E. $=0.5)$ posee más instrumentalidad positiva que la mexicana (M. $=3.8$, D.E. $=0.57)$.

Con relación a la instrumentalidad negativa, hubo efecto significativo de interacción $[\mathrm{F}(1,928)=5.74, \mathrm{p}<0.05]$, efecto principal por lugar de origen $[F(1,928)=60.36, p<0.05] y$ efecto principal por sexo $[\mathrm{F}(1,928)=45.15$, $\mathrm{p}<0.05]$. Los promedios muestran que los hombres $(\mathrm{M} .=2.21$, D.E. $=0.7)$ son más instrumentales negativos que las mujeres
$(\mathrm{M} .=1.92$, D.E. $=0.59)$, y también que los bolivianos $(\mathrm{M} .=2.21$, D.E. $=0.66)$ presentan niveles más altos que los morelenses $(\mathrm{M} .=1.89$, D.E. $=0.62)$. Para el caso de la interacción entre las variables, los hombres bolivianos $(\mathrm{M} .=2.4$, D.E. $=0.69)$ son quienes presentan medias más altas y las mujeres mexicanas $(\mathrm{M} .=1.8$, D.E. $=0.59)$ quienes presentan las medias más bajas.

Por su parte, la expresividad positiva mostró efecto significativo de interacción $[F(1,956)=7.25, \quad p<0.05]$, efecto

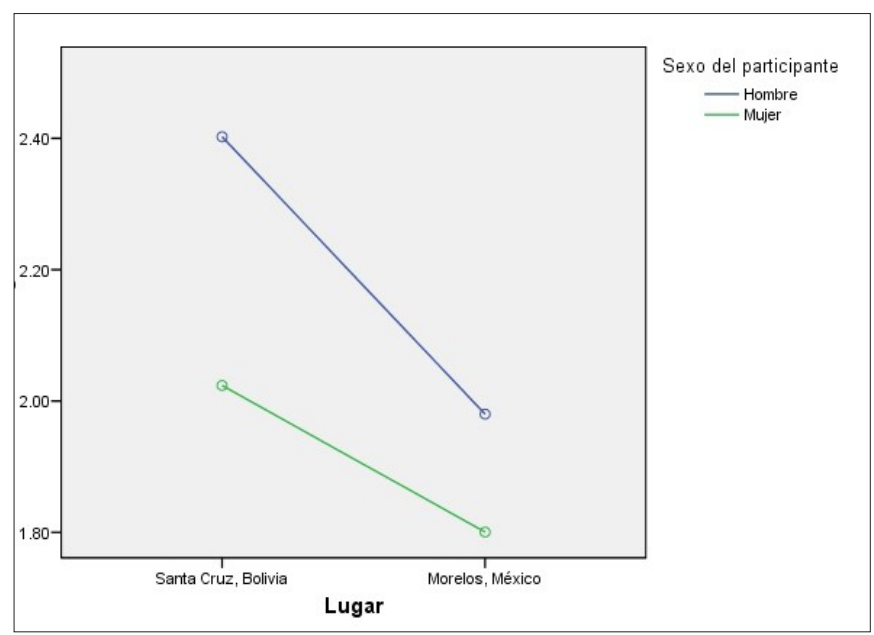

Figura 1. Medias marginales de los grupos sexo x lugar de origen para instrumentalidad negativa. Los puntajes en el eje y son medias estandarizadas.

principal para sexo $[\mathrm{F}(1,956)=77.43$, $\mathrm{p}<0.05]$, pero no mostró efecto principal para lugar de origen $[F(1,956)=3.22$, $\mathrm{p}>0.05]$. Los promedios señalan que las mujeres $(\mathrm{M} .=3.94$, D.E. $=0.66)$ presentan mayores niveles de expresividad positiva que los hombres (M. $=3.56$, D.E. $=0.7)$. En cuanto a la interacción, las mujeres mexicanas son quienes presentan medias más altas $(\mathrm{M} .=3.96$, D.E. $=0.67)$ y son los hombres mexicanos quienes presentan las medias más bajas $(\mathrm{M} .=3.45$, D.E. $=0.74)$.

El último factor de género, Expresividad Negativa, no mostró efecto de interacción entre sexo y lugar de origen $[\mathrm{F}(1,944)=3.69, \mathrm{p}>0.05]$ ni principal de sexo $[F(1,944)=3.02, p>0.05]$. El único efecto significativo fue el principal por lugar de origen $[F(1,944)=33.33, p<0.05]$. Las medias indican que los bolivianos 
$(\mathrm{M} .=2.31$, D.E. $=0.68)$ tienen niveles más altos de expresividad negativa que los mexicanos $(\mathrm{M} .=2.06,0.65)$.

El primer factor de felicidad, llamado satisfacción, no mostró efecto de interacción $\quad[\mathrm{F}(1,901)=0.034, \mathrm{p}>0.05]$, aunque sí principal significativo por lugar de origen $[F(1,901)=14.17, p<0.05]$ y principal por sexo $[\mathrm{F}(1,901)=4.71$,
$[F(1,917)=1.44, p>0.05]$ no fueron significativos. Las medias muestran que los mexicanos (M. $=1.67$, D.E. $=0.69)$ poseen niveles más bajos de infelicidad que los bolivianos (M.=1.8, D.E.=0.8).

Por último, el factor de optimismo no presentó efectos significativos de interacción $\quad[\mathrm{F}(1,946)=1.34, \quad \mathrm{p}>0.05]$ ni efectos principales por sexo

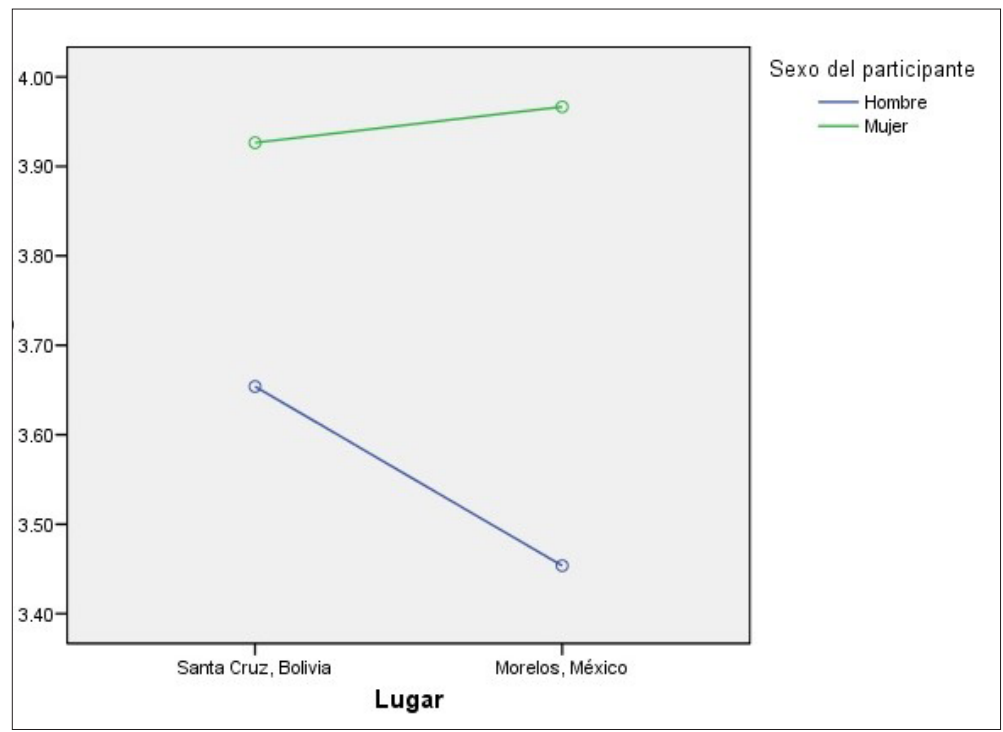

Figura 2. Medias marginales de los grupos sexo x lugar de origen para expresividad positiva. Los puntajes en el eje y son medias estandarizadas.

$\mathrm{p}<0.05]$. Los promedios indican que los bolivianos $(\mathrm{M} .=3.8$, D.E. $=0.65)$ están más satisfechos que los mexicanos $(\mathrm{M} .=3.62$, D.E. $=0.8)$, y que las mujeres $(\mathrm{M} .=3.77$, D.E. $=0.8)$ están más satisfechas que los hombres $(\mathrm{M} .=3.67$, D.E. $=0.7)$.

Para el factor de infelicidad, solo se obtuvo un efecto principal significativo por lugar de origen $[F(1,917)=7.39, p<0.05]$. El efecto principal por sexo $[F(1,917)=1.52$, $\mathrm{p}>0.05]$ y el efecto de interacción
$[\mathrm{F}(1,946)=0.001, \mathrm{p}>0.05]$, pero sí obtuvo efectos significativos principales por lugar de origen $[F(1,946)=12.03, p<0.05]$. Los promedios muestran que los mexicanos (M. $=4.18$, D.E. $=0.79)$ son menos optimistas que los bolivianos (M.=4.34, D.E. $=0.61)$.

Para evaluar la relación entre felicidad y género, se llevó a cabo una correlación producto-momento de Pearson. Para el caso de la muestra boliviana, el factor de 
instrumentalidad positiva presentó una correlación negativa, baja con el factor de infelicidad y correlaciones positivas, y moderadas con satisfacción y optimismo. El factor de instrumentalidad negativa presentó correlaciones negativas, bajas con satisfacción y optimismo, mientras que tuvo una correlación positiva, moderada con el factor de infelicidad. El factor de expresividad positiva presentó correlaciones bajas y directas con satisfacción y optimismo. Por último, el factor de expresividad negativa presentó correlaciones bajas, inversas con satisfacción y optimismo, y una correlación positiva, moderada con infelicidad. Otras correlaciones no fueron significativas.

Para el caso de los mexicanos, el factor de satisfacción presentó correlaciones bajas, directas con instrumentalidad positiva y expresividad positiva, a la vez que presentó correlaciones bajas, inversas con instrumentalidad negativa y expresividad negativa. El factor de infelicidad presentó correlaciones positivas, moderadas con los factores de instrumentalidad negativa y expresividad negativa, y una correlación baja, negativa con el factor de instrumentalidad positiva. Por último, el factor de optimismo presentó correlaciones inversas, bajas con los factores de instrumentalidad negativa y expresividad negativa, además de correlaciones directas con instrumentalidad positiva y expresividad positiva. Otras correlaciones no fueron significativas (ver tabla 1).

También, se llevó a cabo un análisis de correlación entre felicidad y género por sexo. Para el caso de los hombres, la instrumentalidad positiva correlacionó de manera positiva, moderada con

Tabla 1

Correlaciones entre felicidad y género por lugar de origen

\begin{tabular}{llllllllll}
\hline & 1 & 2 & 3 & 4 & 5 & 6 & 7 & Media & D.E. \\
\hline 1. Ins. pos. & - & $-.108^{*}$ & $.301^{* *}$ & $-.237^{* *}$ & $.397^{* *}$ & $-.116^{*}$ & $.406^{* *}$ & 4.008 & 0.5 \\
2. Ins. neg. & -0.083 & - & $-.088^{*}$ & $.757^{* *}$ & -0.074 & $.410^{* *}$ & $-.203^{* *}$ & 2.21 & 0.66 \\
3. Exp. pos. & $.330^{* *}$ & -0.088 & - & $.087^{*}$ & $.264^{* *}$ & 0.052 & $.250^{* *}$ & 3.79 & 0.67 \\
4. Exp. neg. & $-.269^{* *}$ & $.778^{* *}$ & 0.044 & - & $-.125^{* *}$ & $.496^{* *}$ & $-.289^{* *}$ & 2.31 & 0.68 \\
5. Satis. & $.378^{* *}$ & $-.159^{* *}$ & $.178^{* *}$ & $-.244^{* *}$ & - & $-.394^{* *}$ & $.534^{* *}$ & 3.8 & 0.65 \\
6. Infel. & $-.204^{* *}$ & $.341^{* *}$ & 0.008 & $.401^{* *}$ & $-.422^{* *}$ & - & $-.422^{* *}$ & 1.8 & 0.8 \\
7. Optim. & $.361^{* *}$ & $-.098^{*}$ & $.172^{* *}$ & $-.184^{* *}$ & $.515^{* *}$ & $-.459^{* *}$ & - & 4.34 & 0.61 \\
Media & 3.82 & 1.89 & 3.7 & 2.06 & 3.62 & 1.67 & 4.18 & & \\
D.E. & 0.57 & 0.62 & 0.75 & 0.65 & 0.8 & 0.68 & 0.79 & & \\
\hline
\end{tabular}

Nota: la parte superior derecha corresponde a Santa Cruz, Bolivia $(n=583)$ y la parte inferior izquierda corresponde a Morelos, México ( $\mathrm{n}=425$ ). Los factores que corresponden a género son: 1) instrumentalidad positiva; 2 ) instrumentalidad negativa; 3) expresividad positiva, y 4) expresividad negativa. Los que corresponden a felicidad son: 5) satisfacción; 6) infelicidad, y 7) optimismo. *p<0.05, ${ }^{* *} \mathrm{p}<0.01$ 
satisfacción y optimismo; el factor de instrumentalidad negativa tuvo una correlación moderada, directa con el de infelicidad y una correlación baja, negativa con optimismo; el factor de expresividad positiva presentó correlaciones bajas con satisfacción, optimismo e infelicidad; y, por último, el factor de expresividad negativa correlacionó de manera negativa y baja con optimismo, y de forma moderada, directa con infelicidad. Otras correlaciones no fueron significativas. En el caso de las mujeres, la satisfacción correlacionó de manera positiva y baja con expresividad positiva, de manera positiva y moderada con instrumentalidad positiva, y de forma baja e inversa con instrumentalidad negativa $\mathrm{y}$ expresividad negativa. El factor de infelicidad correlacionó de manera negativa, baja con instrumentalidad positiva, y de manera positiva, moderada con instrumentalidad negativa y expresividad negativa. Por último, el factor de optimismo correlacionó de manera positiva y moderada con instrumentalidad positiva, de forma positiva y baja con expresividad positiva, y de manera negativa, baja con instrumentalidad negativa y expresividad negativa. Otras correlaciones no fueron estadísticamente significativas.

\section{Discusión}

Al observar los resultados, puede notarse que de los siete factores involucrados en este estudio (cuatro de género, tres de

Tabla 2

Correlaciones entre felicidad y género para hombres y mujeres

\begin{tabular}{llllllllll}
\hline & 1 & 2 & 3 & 4 & 5 & 6 & 7 & Media & D.E. \\
\hline 1. Ins. Pos. & - & -0.007 & $.356^{* *}$ & $-.153^{* *}$ & $.367^{* *}$ & -0.067 & $.395^{* *}$ & 3.89 & 0.53 \\
2. Ins. Neg. & -0.063 & - & 0.075 & $.807^{* *}$ & 0.001 & $.436^{* *}$ & $-.095^{*}$ & 2.21 & 0.7 \\
3. Exp. Pos. & $.277^{* *}$ & $-.113^{*}$ & - & $.167^{* *}$ & $.242^{* *}$ & $.120^{*}$ & $.277^{* *}$ & 3.56 & 0.7 \\
4. Exp. & & & & & & & & & \\
Neg. & $-.269^{* *}$ & $.740^{* *}$ & 0.014 & - & -0.068 & $.497^{* *}$ & $-.163^{* *}$ & 2.24 & 0.73 \\
5. Satis. & $.423^{* *}$ & $-.144^{* *}$ & $.178^{* *}$ & $-.248^{* *}$ & - & $-.307^{* *}$ & $.549^{* *}$ & 3.67 & 0.7 \\
6. Infel. & $-.210^{* *}$ & $.320^{* *}$ & -0.021 & $.412^{* *}$ & $-.467^{* *}$ & - & $-.416^{* *}$ & 1.78 & 0.77 \\
7. Optim. & $.389^{* *}$ & $-.151^{* *}$ & $.169^{* *}$ & $-.260^{* *}$ & $.513^{* *}$ & $-.428^{* *}$ & - & 4.27 & 0.67 \\
Media & 3.95 & 1.92 & 3.94 & 2.16 & 3.77 & 1.71 & 4.26 & & \\
D.E. & 0.55 & 0.59 & 0.66 & 0.63 & 0.76 & 0.74 & 0.72 & & \\
\hline
\end{tabular}

Nota: la parte superior derecha corresponde a Santa Cruz, Bolivia $(n=583)$ y la parte inferior izquierda corresponde a Morelos, México ( $\mathrm{n}=425)$. Los factores que corresponden a género son: 1) instrumentalidad positiva; 2) instrumentalidad negativa; 3) expresividad positiva, y 4) expresividad negativa, y los factores que corresponden a felicidad son: 5) satisfacción; 6) infelicidad, y 7) optimismo. ${ }^{*} \mathrm{p}<0.05,{ }^{*} \mathrm{p}<0.01$ 
felicidad), todos tienen un efecto directo o de interacción por lugar de origen, lo que podría atribuir las diferencias a la ubicación geográfica y su inherente carga cultural; y solo tres presentan efectos directos por sexo de las personas.

Congruente con los trabajos de Vergara (2002), los puntajes de los hombres son más altos en instrumentalidad (masculinidad) mientras que los de las mujeres son más altos en expresividad (feminidad), resultado que parece acentuarse en México a partir de la significancia estadística de los datos y que perpetúa, en parte, lo originalmente planteado por Bem (1993), de que los hombres son masculinos y las mujeres son femeninas. Vergara (2002) hace hincapié en que, a pesar de que las naciones latinoamericanas pueden ser típicamente catalogadas como culturas colectivistas, hay algunas (Argentina, Venezuela, México, Brasil, Colombia y Chile) en las que la instrumentalidad y expresividad tienen un efecto particular ligado al mayor desarrollo socioeconómico, acentuando las diferencias en dimensiones de masculinidad y feminidad entre hombres y mujeres (Damji y Lee, 1995; MurphyBerman, Berman, Singh y Pandy, 1992).

Para el caso de la satisfacción, congruente con lo planteado por Wilson (1967) hace casi medio siglo y corroborado más recientemente por otras investigaciones (Diener, 2009; Fujita et al., 1991; Pozos, 2012; Velasco, 2015), las diferencias por sexo no son definitivas, aunque parecen favorecer a las mujeres. Factores sociales, como los mencionados por Triandis (2000) y Riquelme (2013), estarían favoreciendo un cambio cultural y apertura de pensamiento, lo cual podría estar jugando a favor de las mujeres, aunque con diferencias apenas notables.

Respecto al efecto de la cultura sobre las dimensiones de masculinidadfeminidad, vale la pena hacer notar que las medias son muy similares en ambas muestras, tal como reportan Fernández, Zubieta y Páez (2000), quienes afirman que al evaluar estas dimensiones, las diferencias son relativas, ya que aún entre las culturas colectivistas la media informada se acerca a lo típico o frecuente. Sin embargo, las diferencias, aunque apenas notables, podrían deberse a otros factores.

Bolivia, junto con Perú y Guatemala, son considerados países con alta proporción de población indígena (Fernández et al., 2000); mientras que México se ubica en un nivel intermedio. Esta proporción podría estar moderando la vivencia y expresión de emociones positivas y negativas junto con el desarrollo económico mencionado anteriormente. También, México es considerada una cultura muy masculina, y tal como proponen Díaz Loving et al. (2012), los extremos instrumentales o expresivos se asocian con mayores déficits en la expresión verbal de las emociones y menor eficacia en la comunicación directa, lo que podría explicar un mejor ajuste en la muestra boliviana.

Según las investigaciones de Hofstede (1991, 1998) y Fernández et al. (2000), las culturas nacionales (cada nación se entiende como una cultura independiente) latinoamericanas son típicamente 
colectivistas, lo que colocaría a las dos muestras de este trabajo en igualdad de condiciones: un cuadro social de estructura cerrada al seno del cual los individuos son implicados emocionalmente, como una familia extendida, un grupo social, o cualquier grupo que brinde protección (Hofstede, 1998). Sin embargo, los datos de Fernández et al. (2000) ubican a México como una cultura colectivista limítrofe, esto es, con tintes individualistas, en donde la conducta tiende a ser más egocéntrica. México, de un total de veinte países (latinoamericanos y europeos), se ubica en el grupo de países individualistas que considera a naciones europeas (Alemania, Suiza, España, Francia) y a Estados Unidos, que se coloca como el país más individualista de toda la muestra.

Comparativamente, los hallazgos de Fernández et al. (2000) ubican a Bolivia como una nación más femenina que México, lo que implica que hay mayor probabilidad de encontrar roles sociales sexuales muy imbricados en los cuales tanto el rol femenino como el masculino se caracterizan por la necesidad de una relación más expresiva, por la modestia, la preocupación por los demás y la importancia dada a la calidad no material de la vida. Países como México responden al tipo de sociedades en las que el rol masculino se caracteriza por la necesidad de realización, la asertividad, el uso de la fuerza y la importancia dada al éxito material.

Aún así, existen investigaciones (Mesquita y Frijda, 1992; Zubieta,
Fernández, Vergara, Martínez y Frijda, y Candia 1998) que defienden con base en evidencia empírica la idea de la relativa similitud transcultural que existe en dimensiones como las de las expresiones de las emociones y manifestación de ciertas conductas vinculadas al género (reacciones verbales, faciales, procesos de regulación, enfrentamiento) fuertemente relacionados con la variabilidad cultural. Investigaciones como las de Zubieta et al. (1998) adjudican las diferencias mínimas existentes entre Estados Unidos y el resto de América Latina al carácter individualista y colectivista, respectivamente. Esta diferencia explica en muchos casos las diferencias en estilos de pensamiento, en filosofía de vida, cooperación, competición, defensa de intereses y opiniones individuales, cercanía física, entre otras dimensiones. Por lo tanto, vale la pena tomar en cuenta la cercanía de México con un país como Estados Unidos, que es considerado como un país altamente individualista y masculino (Fernández et al., 2000; Hofstede, 2001; Vergara, 2002). Esta similitud entre México y Estados Unidos es congruente con los hallazgos de Frías, Shaver y Díaz Loving (2013), y podría estar moderando la expresión de rasgos instrumentales y expresivos típicos mexicanos (Díaz Loving, 2005) a raíz de un proceso de aculturación, haciendo que la muestra mexicana no se comporte tan colectivamente como podría pensarse inicialmente.

Veenhoven (2012a) afirma que la felicidad es un constructo social, por lo 
que depende de nociones compartidas sobre la vida que encuadran después evaluaciones individuales. Inicialmente, la premisa planteada por Tov y Diener (2009) parece cumplirse: existen diferencias en niveles de felicidad entre culturas. Sin embargo, las diferencias en los niveles de los tres factores de felicidad no son notables, congruente con lo planteado por Veenhoven (2012a, 2012b), quien afirma que de ser cierto que la felicidad tiene componentes culturalmente específicos fundamentales las diferencias deberían ser considerables y no deberían mostrar similitud "universal".

Las diferencias en los factores de felicidadpueden deberse también a las diferencias de individualismo-colectivismo exploradas anteriormente (Helliwell, Huang y Wang; 2014). Como ya se mencionó, la ligera inclinación de México hacia el individualismo (Fernández et al., 2000; Frías et al., 2013; Hofstede, 2001) y el colectivismo tradicional de Bolivia podrían explicar las mínimas pero significativas diferencias. Las diferencias en las medias de niveles de felicidad podrían deberse a que Bolivia sigue siendo y México está dejando de ser prosocial, orientado al contacto social, al trabajo colectivo, a la recompensa indirecta a través de la ganancia de terceras personas, etcétera.

Estas diferencias entre individualismocolectivismo también se ven reflejadas en un sentido de identidad individual e identidad grupal, respectivamente, que tiene impacto en el grado con que las personas se evalúan en términos de felicidad. Específicamente, las pequeñas diferencias en los niveles de felicidad podrían deberse a que, en países colectivistas, esta no depende solamente de alcanzar metas propias, sino también del cumplimiento de estándares sociales, contribuir al bienestar común y al éxito individual sumado al grupal (Helliwell et al., 2014). Parecería entonces que la postura de Diener y Diener (1996), respecto a las culturas en las que el colectivismo está más marcado, como es el caso de Bolivia, sostiene que los individuos efectivamente socializados a tieneden a expresarse y actuar de manera feliz.

En lo que se refiere a las correlaciones entre los factores de género y felicidad, en ambos casos (correlaciones por sexo y por lugar de origen) los análisis muestran patrones similares: los rasgos positivos de instrumentalidad y expresividad están asociados con satisfacción y optimismo, y los rasgos negativos con infelicidad. Múltiples investigaciones (Bassof y Glass, 1982; Díaz-Loving et al., 2007, 2012; Hegelson y Fritz, 1999; Matud et al., 2002; Pozos, 2012; Sanjuán y Jensen, 2013; Sanjuán y Magallares, 2013; Velasco, 2015) han obtenido resultados similares a los de la presente investigación: Siempre que los individuos estén orientados hacia su propia cultura, e incluso cuando exista una apertura de pensamiento (sin que llegue a ser radical), la asociación entre felicidad y género será tal como se presenta en este trabajo, tanto para hombres como mujeres. 
El impacto que tienen los rasgos instrumentales y expresivos positivos en hombres y mujeres de Bolivia y México es favorable en tanto que existen factores como la cooperación, la orientación al logro, el control y expresión de las emociones, la presencia de bajos niveles de ansiedad y depresión, bajo egocentrismo, respeto, el cuidado de los otros, la racionalidad, el pensamiento lógico y asertividad, entre otros, que promueven un sentimiento de valía personal, de estabilidad, satisfacción, sentido positivo de la vida y desarrollo personal.

A pesar de los resultados aquí expuestos y discutidos, las variaciones culturales asociadas al lugar de origen de una persona y el sexo biológico de esta parecen no ser determinantes en la expresión de ciertos rasgos de género y niveles de felicidad, así como tampoco parece ser determinante la interacción de estas dos variables. La interrelación de variables por sexo biológico y lugar de origen parece confirmar que existen más similitudes que diferencias entre las dos muestras de este estudio, lo que podría explicar las diferencias mínimas entre mexicanos y bolivianos. Tal como mencionan Alarcón $(2000,2006)$ y Biswas-Diener et al. (2009), la expresión de ciertas características de personalidad puede estar determinada por diversos factores culturales, bienes materiales, éticos, estéticos, psicológicos, religiosos y otros recursos sociales, por lo que se recomienda incluir otras variables en el estudio de la instrumentalidad, expresividad y felicidad a futuro.

\section{RefERENCIAS}

Alarcón, R. (2000). Variables psicológicas asociadas con la felicidad. Persona, 3, 147-157.

Alarcón, R. (2002). Fuentes de felicidad: ¿Qué hace feliz a la gente? Revista de Psicología de la PUCP, XX(2), 171-196.

Alarcón, R. (2006). Desarrollo de una Escala Factorial para Medir la Felicidad, Revista Interamericana de Psicología, 40(1), 99-106.

Allen-Kee, D. (1980). The relationship of psychological androgyny to self-esteem and life satisfaction in career-oriented and home-oriented women (Doctoral dissertation, University of Southern California, 1980). Dissertation Abstracts International, 41, 1479A.

Andrews, F. M., y Withey, S. B. (1976). Social indicators of wellbeing: America's perception of life quality. New York: Plenum Press.

Bassof, E. S., y Glass, G. V. (1982). The relationship between sex roles and mental health: A meta-analysis of twenty six studies. The Counseling Psychologist, 10, 105-112. 
Bem, S. L. (1981). Gender Schema Theory: A cognitive account of sex-typing. Psychological Review, 88, 354-364.

Bem, S. L. (1993). The lenses of gender: transforming the debate on sexual inequality. Yale University.

Biswas-Diener, R., Vitterso, J., y Diener, E. (2009). Most People are Pretty Happy, but There is Cultural Variation: The Inughuit, the Amish, and the Maasai. En E. Diener (Ed.), The Science of Well Being: The Collected Works of Ed Diener, Social Indicators Research Series, 38, 245-260.

Campbell, K. K. (1993). Men, women and agression. New York: Basic Books.

Damji, T., y Lee, C.M. (1995). Gender role identity and perceptions of Ismaili Muslim men and women. Journal-of-Social-Psychology, 135(2), 215-223.

Díaz-Loving, R. (2005). Emergence and contributions of a Latin American indigenous social psychology. International Journal of Psychology, 40, 213-227.

Díaz-Loving, R., Rocha, T., y Rivera, S. (2007). La instrumentalidad y expresividad desde una perspectiva psico-socio-cultural. México: Porrúa.

Díaz-Loving, R., y Rocha, T. (2008). La masculinidad y la feminidad a través del ciclo vital: un estudio comparativo. En: S. Rivera Aragón, R. Díaz Loving, R. Sánchez Aragón e I. Reyes Lagunes (Eds.), La psicología social en México, XII (pp. 213-220), México: AMEPSO.

Díaz-Loving, R., Rivera Aragón, S., y Velasco Matus, P. W. (2012). Masculinidad-feminidad y salud mental. Persona, 15, 137-157.

Diener, E. (2005). Guidelines for National Indicators of Subjective Well-being and Illbeing. Illinois: University of Illinois.

Diener, E. (2009). The Science of Well Being: Reviews and Theoretical Articles. En E. Diener (Ed.), The Science of Well Being: The Collected Works of Ed Diener, Social Indicators Research Series, 37, 1-10.

Diener, E. y Diener, C. (1996). Most people are happy. Psychological Science, 7, 181-185.

Diener, E., Suh, E. M., Lucas, R., y Smith, H. (1999). Subjective Well-being: Three Decades of Progress. Psychological Bulletin, 125(2), 276-302.

Diener, E., y Diener, M. (2009). Cross-Cultural Correlates of Life Satisfaction and SelfEsteem. En E. Diener (Ed.), The Science of Well Being: The Collected Works of Ed Diener, Social Indicators Research Series, 38, 71-91.

Fernández, J. (1996). Varones y mujeres: desarrollo de la doble realidad del sexo y del género. Madrid, España: Psicología Pirámide. 
Fernández, I., Zubieta, E., y Páez, D. (2000). Expresión e inhibición emocional en diferentes culturas. En D. Páez y M. M. Casullo (Comps.), Cultura y alexitimia: ¿cómo expresamos aquello que sentimos? (pp. 73-98). Buenos Aires: Paidós.

Frías, M., Shaver, P., y Díaz Loving, R. (2013). Individualism and collectivism as moderators of the association between attachment insecurities, coping, and social support. Journal of Social and Personal Relationships, 1-29.

Fujita, F., Diener, E., y Sandvik, E. (1991). Gender differences in negative affect and well-being: The case for emotional intensity. Journal of Personality and Social Psychology, 61, 427-434.

Green, B. L. y Kendrick, D. T. (1994). The attractiveness of gender-typed traits at different relationship levels: Androgynous characteristics may be desirable after all. Personality and Social Psychology Bulletin, 20, 244-253.

Hegelson, V. S., y Fritz, H. L. (1999). Unmitigated agency and unmitigated communion: Distinctions from agency and communion. Journal of Research in Personality, 33, $133-158$.

Heilbrun, A. B. (1981). Human sex-role behavior.Nueva York: Pergamon Press.

Helliwell, J., Huang, H., y Wang, S. (2014). Social capital and well-being in times of crisis. Journal of Happiness Studies, 15, 145-162.

Hofstede, G. H. (1998). Masculinity and Feminity: The taboo dimension of national cultures. Beverly Hills, CA: Sage.

Hofstede, G. H. (1980). Culture's consequences: International differences in workrelated values. Beverly Hills, CA: Sage.

Hofstede, G. H. (1991). Culture and organizations: Software of the mind. London: McGraw-Hill.

Hofstede, G. H. (2001). Culture's consequences: Comparing values, behaviors, institutions, and organizations across nations. Thousand Oaks, CA: Sage.

Jayme, M., y Sau, V. (2004). Psicología diferencial del sexo y el género. España: Icara.

Kozma, A. y Stones, M. J. (1983). Predictors of happiness. Journal of Gerontology, 38, 626-628.

Matud, A. M. P., Rodríguez, W. C., Marrero, Q. R., y Carballeira, A. M. (2002). Psicología del género: implicaciones en la vida cotidiana. Madrid: Biblioteca nueva.

Mesquita, B., y Frijda, N. H. (1992). Cultural variations in emotions: A review. Psychological Bulletin, 112, 179-204.

Moen, P., Robinson, J., y Dempster, M. D. (1995). Caregiving and women's well-being: A life course approach. Journal of Health and Social Behavior, 36(3), 259-273. 
Murphy-Berman, V. A., Berman, J. J., Singh, P., y Pandy, J. (1992). Cultural variations in sex typing: A comparison of students in the United States, Germany, and India. Journal-of-Social-Psychology, 132(3), 403-405.

O'Sullivan, W. M. (1980). A study of the relationship between life satisfaction of the aged and perceptions they hold concerning their own masculinity or femininity (Doctoral dissertation, New York University, 1980). Dissertation Abstracts International, 41, 6745B. (University Microfilms No. 80-17,520).

Palomar, J. (2005). Estructura del bienestar subjetivo: Construcción de una escala multidimensional. En: L. Garduno, B. Salinas y M. Rojas (Coords.), Calidad de vida y bienestar subjetivo en México (pp. 113-142), México: Plaza y Valdés S.A. de C.V.

Pozos Gutiérrez, J. L. (2012). Etnopsicología de la felicidad en la pareja. Tesis de doctorado no publicada. México: Universidad Nacional Autónoma de México, Facultad de Psicología.

Riquelme, A. M. (2013). Factores psicosociales que afectan la relación de pareja cuando la mujer tiene un crecimiento profesional y económico mayor que el del hombre. Tesis de doctorado no publicada. México: Universidad Nacional Autónoma de México, Facultad de Psicología.

Rocha, T. (2004). Socialización y cultura: El impacto de la diferenciación entre los sexos. Tesis inédita de Doctorado. México: Universidad Nacional Autónoma de México, Facultad de Psicología.

Rocha, T. (2008). Identidad de género y estado anímico en hombres y mujeres. En: S. Rivera Aragón, R. Díaz Loving, R. Sánchez Aragón e I. Reyes Lagunes (Eds.), La psicología social en México, XII (pp. 663-670). México: AMEPSO.

Sanjuán, P., y Jensen, K. (2013). Relationships between self-serving attributional bias and subjective well being among Danish and Spanish women. In H. Knoop y A. Delle Fave (Eds.), Well-being and cultures: Perspectives from positive psychology (pp. 183194). Dordrecht: Springer.

Sanjuán, P., y Magallares, A. (2013). Coping strategies as mediating variables between self-serving attributional bias and subjective well being. Journal of Happiness Studies, 15, 443-453.

Spence, J. (1993). Gender-Related Traits and Gender Ideology: Evidence for a Multifactorial Theory. Journal of Personality and Social Psychology, 64 (4), 624-635.

Suh, E., Diener, E. y Updegraff, J. (2008). From Culture to Priming Conditions: SelfConstrual Influences on Life Satisfaction Judgements, Journal of Cross Cultural Psychology, 39(3), 3-15. 
Tov, W., y Diener, E. (2009). The Well-being of Nations: Linking Together Trust, Cooperation, and Democracy. En Diener (Ed.), The Science of Well Being: The Collected Works of Ed Diener, Social Indicators Research Series, 37, 155-173.

Triandis, H. C. (2000). Dialectics between cultural and cross-cultural psychology. Asian Journal of Social Psychology, 3, 185-195

Veenhoven, R. (2005). Lo que sabemos de la felicidad. En: L. Garduno, B. Salinas y M. Rojas (Coords.), Calidad de vida y bienestar subjetivo en México (pp. 17-56), México: Plaza y Valdés.

Veenhoven, R. (2012a). Cross-national differences in happiness: Cultural measurement bias of effect of culture? International Journal of Wellbeing, 2(4), 333-353.

Veenhoven, R. (2012b). Correlates of Happiness. World Database of Happiness. Erasmus University.

Velasco, P. (2015). Una aproximación bio-psico-sociocultural al studio del bienestar subjetivo en México: Un modelo explicativo/predictivo. Tesis de doctorado no publicada. México: Universidad Nacional Autónoma de México, Facultad de Psicología.

Vergara, A. I. (2002). Autoconcepto de género y variabilidad cultural en América. Revista Internacional de Ciencias Sociales y Humanidades, SOCIOTAM, XXI, 47-62.

Wilson, W. R. (1967). Correlates of avowed happiness. Psychological Bulletin, 67, 294-306.

Zubieta, E., Fernández, I., Vergara, A., Martínez, M. D., y Candia, L. (1998). Cultura y emoción en América. Boletín de Psicología, 61, 65-89. 